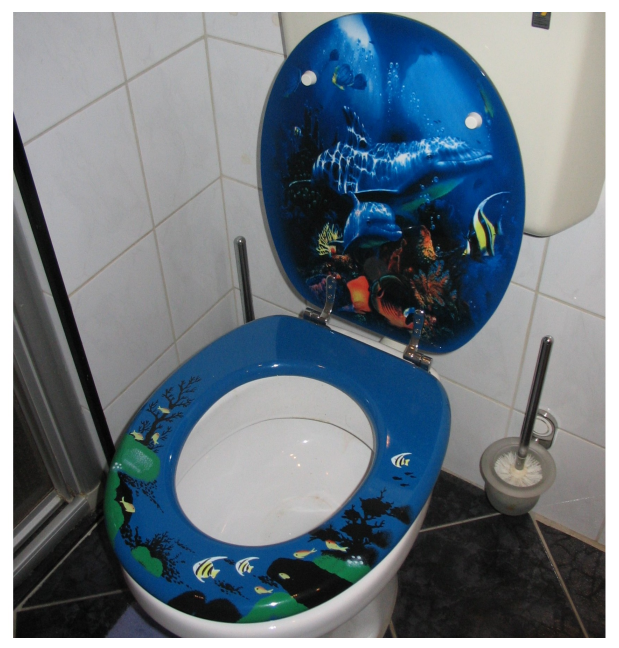

\title{
Covid-19: A fecal-orally transmitted disease with severe involvement of the lungs
}

Carlos Rodriguez

1 Fundación Jiménez Díaz

\section{Abstract}

Undoubtely, the more salient feature, and the main cause of death, of the Covid-19 disease is its pulmonary involvement, the so-called Acute Respiratory Distress

Syndrome or ARDS. The fact that the cause of the 2003 SARS outbreak, the SARS-

CoV1 virus, also induced a predominantly pulmonary disease, has put the focus on the lung, often overlooking one theme that is stubornly present at least in the Covid-19 disease: the viruses are isolated in the feces of patients (syntomatic or asymptomatic alike) much after the nasopharyngeal swabs become negative.

Here I present evidence strongly suggesting that, besides airborne spreading, the main transmission mode of the SARS-Cov-2 virus is of the faecal-oral type. I hypothesize that, in the near future, the Covid-19 disease could be silently transmitted through a predominantly faecal-oral mechanism. This model is based in the more than probable existence of an intestinal reservoir in convalescent and, more conspicuously, in the likely large cohort of asymptomatic carriers. 
The implications of the study are broad and should prompt Health Authorities to planify the best possible ways to carry thoroughly designed programs to analize and curtail this mode of viral perpetuation and transmission.

\section{THE INTESTINAL VIRUS}

The closest relative of the SARS-Cov2 virus is the endogenous bat coronavirus RaT G13 (96.2\% nucleotide homology). Much like the Cov2 in many humans, the RaT G13 does not cause symptoms in bats but is believed to cause intra and inter-species spread through droppings ${ }^{[1]}$. Importantly, as we will see below, the bat virus shares the same receptor on infected cells as both human SARS-Cov and SARS-CoV2 ${ }^{[2]}$.

For the last two months we have been reading about something that at the beginning sounded like a curious phenomenon: the frequent positivity for the SARS-Cov2 virus in fecal samples of asymptomatic contacts of people with the disease, or in covid-19 patients long after being negative for the virus in pharyng eal swabs. Indeed, in a first published series, the fecal samples remained positive in half of the patients for a mean of 11.2 days after the respiratory tract samples became negative for SARS-CoV-2 RNA ${ }^{[3]}$. In another study, $64.29 \%$ of patients remained positive for viral RNA in the feces after the pharyngeal swabs turned negative for an average period of 7 (6-10) days ${ }^{[4]}$. Interestingly, a recent case report showed that 3 out of 5 asymptomatic household contacts of a physician with Covid-19 disease tested positive for SARS-CoV-2 on stool specimens ${ }^{[5]}$. Finally, in the more recent patient series, duration of faecal viral shedding ranged from 1 to 33 days after nasopharyngeal swab turned neg ative with one result remaining positive after 47 days of onset of symptoms ${ }^{[6]}$.

Together these results suggest that the virus is actively replicating in the patient's gastrointestinal tract beyond what was believed to be the usual period of the initial infection. This very possibility has raised concerns among specialists allerted about the public health issues that these findings imply for the future management of the disease ${ }^{[7]}$

But before we enter into a discussion about the meaning of those findings, we should be reminded that the periods of fecal shedding reported so far are probably still not definitive. Indeed, it should be pointed out that due to the recentness of the first disease cases and the time of preparation of manuscripts, the reported follow-ups in the referred 
publications can't be possibly longer than two months, which, not surprisingly, is approximately the longest follow-up period reported. This is why I forsee that, in the near future, we will be witnessing positive fecal PCR tests, months and even years after passing the disease or, more often than previouly thought, after an unreported asymptomatic infection.

\section{THE ACE2 RECEPT OR AS THE INTESTINAL CUE FOR THE SARS-COV-2 VIRUS}

Although it seems that there could be alternative receptors, it is beyond doubt that the main SARS-Cov2 receptor on human cells is the hACE2 protease. This protein has been found to be expressed in many human tissues. After a thorough mining of the GTEx repository of human tissues, it has been reported that the small intestine, testis, kidneys, heart, thyroid, and adipose tissue had the highest ACE2 expression levels. Importantly, in this study, the small intestine was, by far, the tissue with the highest expression level among the 31 different tissues analized. Of note, the expression level of ACE2 in the lung lagged far behind those tissues (about one order of magnitude less expression than the intestine ${ }^{[8]}$. This alone would suggests that the SARS-CoV2 virus could have a preference for infecting the small intestine. Whether the intestinal infected cells are epithelial, immune or mesenchymal remains to be elucidated.

In addition to ACE2, the SARS-COV2 virus, in order to achieve an efficient cell entry and infectivity rate, needs the action of another cell surface protease (The transmembrane Serine Protease 2 or TMPRSS2) on the virus Spike protein. Interestingly, TMPRSS2 is relatively highly expressed along most of the intestinal tract ${ }^{[9]}$, making the gut a perfect terrain for viral survival and replication.

Finally, although the SARS-CoV2 virus has turned out to have a much higher infectivity rate (i. E. more contagious) than its close relative, the SARS-CoV-1 (the cause of the 2003 SARS outbreak), the stability of both viruses seems to be similar under very different experimental settings, excluding different extracorporeal survival rates as a plausible explanation. This indicates that, as has been pointed out, differences in the epidemiologic characteristics of these viruses probably arise from other factors ${ }^{[10]}$. Among these factors, one that now comes into play is fecal viral shedding. Although the SARS-CoV-1 virus also seems to be eliminated through feces ${ }^{[11]}$, it is not excluded that a major difference in fecal viral load or fecal viral viability could account for the differences in infectivity rates between the two viruses ${ }^{[12]}$. Interestingly, the other coronavirus causing a severe disease in humans, the MERS-Cov virus, does not use ACE2 as a 
doorway to enter human cells, and importantly, is only rarely present in the feces of camels that are otherwise positive for the virus in nasal swabs ${ }^{[13]}$, suggesting that the high expression levels of the ACE2 protein in the human intestine could account for the different infectious behaviors between those viruses.

These findings alone highlight the importance of the ACE2 protease not only in the physiopathology of the covid-19 related ARDS, but, importantly, in the infection of human intestinal epithelial cells and the maintenance of the infectious intestinal niche of the disease.

\section{INTESTINAL NICHES ARE A COMMON THEME IN VIRAL INFECTIONS}

Viral tissue reservoirs have been described for other viruses. These viral niches provide a mechanism for chronic infective conditions that are often refractory to treatments aimed at viral eradication. For instance, the persistence of HIV in tissue reservoirs remains a major obstacle to eradicating HIV in infected patients and it has been elegantly shown that the large intestine is a major viral reservoir in Long Term Non-progressive infection (LTNPs) variants of the disease ${ }^{[14]}$. Also, 33\% of stool samples from 179 children aged 0-6 years attending Danish day care centers tested positive for at least one gastrointestinal virus although no gastrointestinal symptoms were reportedly observed during the preceding two months for most $(n=144,63 \%)$ of the positive samples ${ }^{[15]}$. Additionally, the gastrointestinal mucosa can be an extrahepatic reservoir of the Hepatitis C Virus (HCV) and that could contribute to viral recurrence after liver transplantation ${ }^{[16]}$.

To summarize, so far, I have shown that the human coronavirus SARS-CoV2, the causative agent of the Covid-19 disease, may have a preference to infect the human intestine due, at least in part, to the high expression levels of its human receptor, the ACE2 protein. By infecting cells in the intestinal wall, it is likely to perpetuate itself long after the initial infection, thus becoming a potential source of continuous spreading.

Incidentally, this intestinal reservoir could also provide an explanation for the small number of cases of so-called "reinfection" based on positive retesting on nasopharyngeal or rectal swabs after previous double negative testing ${ }^{[17]}$. The main argument in response to the re-infection thesis seems to be an early report showing that reinfection could not occur in SARS-CoV-2 infected rhesus macaques ${ }^{[18]}$. In a humanly understandable effort to dispel the possibility of a potential viral re-infection, many are 
sticking to this paper or, worst, to farfetched theories, as a proof that something like a reinfection is scientifically unsound in such a short period of time. However reality is more stubborn than theories and I believe that there is a different explanation for this apparently inexplicable finding. In fact, current evidence sustains the idea that what looks like a re-infection could actually reflect the existence of a continuous spread of the virus from an intestinal reservoir. To an untrained observer, a positive PCR test could seem as a reinfection when it could actually reflect different viral replication rates in an individual that never really was virus-free.

Finally, in the last few weeks, as a practicing pediatrician in Madrid, I have been witnessing outbreaks of unusual symptoms (intestinal, cutaneous, protracted cough, etc.) in well kept and isolated kids living with asymptomatic parents, most of them staying at home for more than 6 weeks. This alone should raise the question as to how in a confined asymptomatic environment these symptoms can arise. A now likely explanation may lie in the possibility that one (or both) of the parents be a silent spreader of the virus from his/her intestinal viral niche.

In summary, hereby I present evidence strongly suggesting that a major mode of transmission of the SARS-Cov-2 virus is of the faecal-oral type. I hypothesize that, in the near future, the Covid-19 disease could be silently transmitted through a predominantly faecal-oral mechanism. This model is based, much like in bats, in the more than probable existence of an intestinal reservoir in convalescent and, more conspicuously, in the likely large cohort of asymptomatic viral spreaders.

No doubt, there is an airborne mode of transmission in Covid-19 disease and we should all be aware of the basic precautions to be taken to avoid this route of trasmission. By now everybody is aware of terms such as "social distancing" or "N95 masks". I believe that, from now on, the main emphasis should be put in the mode of that threatens to be the chosen way by the virus to expand and perpetuate itself around the globe: the fecaloral dissemination.

Since preparedness is key to survival, we should thus brace ourselves for the possibility, as improbable as we think it could be, of a protracted pervasive way of viral penetration in broad populations, through the silent continuous spread of viable viruses in human feces. 


\section{References}

1. ^ L. L. M. Poon, D. K. W. Chu, K. H. Chan, O. K. Wong, T. M. Ellis, Y. H. C. Leung. (2005). Identification of a Novel Coronavirus in Bats. JVI, vol. 79 (4), 2001-2009. doi:10.1128/jvi.79.4.2001-2009.2005.

2. ' Zhenglin Zhu, Kaiwen Meng, Geng Meng. (2020). The genomic recombination events may reveal the evolution of coronavirus and the origination of 2019-nCoV. doi:10.21203/rs.3.rs-21488/v1.

3. `Yongjian Wu, Cheng Guo, Lantian Tang, Zhongsi Hong, Jianhui Zhou, Xin Dong. (2020). Prolonged presence of SARS-COV-2 viral RNA in faecal samples. The Lancet Gastroenterology \& Hepatology, vol. 5 (5), 434-435. doi:10.1016/s24681253(20)30083-2.

4. ` Yifei Chen, Liangjun Chen, Qiaoling Deng, Guqin Zhang, Kaisong Wu, Lan Ni. (2020). The presence of SARS-COV-2 RNA in the feces of COVID-19 patients.J Med Virol. doi:10.1002/jmv.25825.

5. ^ Yi Luo, Edwin Trevathan, Zhengmin Qian, Yirong Li, Jin Li, Wei Xiao. (2020). Asymptomatic SARS-CoV-2 Infection in Household Contacts of a Healthcare Provider, Wuhan, China. Emerg. Infect. Dis., vol. 26 (8). doi:10.3201/eid2608.201016.

6. ^ Sunnia Gupta, Jody Parker, Stephanie Smits, Jonathan Underwood, Sunil Dolwani. (2020). Persistent viral shedding of SARS-CoV-2 in faeces - a rapid review. doi:10.1101/2020.04.17.20069526.

7. ^ E. Susan Amirian. (2020). Potential Fecal Transmission of SARS-CoV-2: Current Evidence and Implications for Public Health. International Journal of Infectious Diseases. doi:10.1016/j.jijd.2020.04.057.

8. ' Meng-Yuan Li, Lin Li, Yue Zhang, Xiao-Sheng Wang. (2020). Expression of the SARSCoV-2 cell receptor gene ACE2 in a wide variety of human tissues. Infect Dis Poverty, vol. 9 (1). doi:10.1186/s40249-020-00662-x.

9. ^ PeterJ. Thul, Cecilia Lindskog. (2017). The human protein atlas: A spatial map of the human proteome. Protein Science, vol. 27 (1), 233-244. doi:10.1002/pro.3307.

10. ^ Neeltje van Doremalen, Trenton Bushmaker, Dylan H. Morris, Myndi G. Holbrook, Amandine Gamble, Brandi N. Williamson. (2020). Aerosol and Surface Stability of SARSCoV-2 as Compared with SARS-CoV-1. N EnglJ Med, vol. 382 (16), 1564-1567. doi:10.1056/nejmc2004973.

11. ' JSM Peiris, CM Chu, VCC Cheng, KS Chan, IFN Hung, LLM Poon. (2003). Clinical progression and viral load in a community outbreak of coronavirus-associated SARS pneumonia: a prospective study. The Lancet, vol. 361 (9371), 1767-1772. 
doi:10.1016/s0140-6736(03)13412-5.

12. ^ S. S. Y. Wong, K.-Y. Yuen. (2008). The management of coronavirus infections with particular reference to SARS. Journal of Antimicrobial Chemotherapy, vol. 62 (3), 437441. doi:10.1093/jac/dkn243.

13. ^ Maged G. Hemida, DanielK.W. Chu, Leo L.M. Poon, Ranawaka A.P.M. Perera, Mohammad A. Alhammadi, Hoi-yee Ng. (2014). MERS Coronavirus in Dromedary Camel Herd, Saudi Arabia. Emerg. Infect. Dis., vol. 20 (7). doi:10.3201/eid2007.140571.

14. ^ Binhua Ling, Mahesh Mohan, Andrew A. Lackner, Linda C. Green, Preston A. Marx, Lara A. Doyle. (2010). The Large Intestine as a Major Reservoir for Simian Immunodeficiency Virus in Macaques with Long-Term, Nonprogressing Infection.J INFECT DIS, vol. 202 (12), 1846-1854. doi:10.1086/657413.

15. ^ Betina Hebbelstrup Jensen, Pikka Jokelainen, Alex Christian Yde Nielsen, Kristina Træholt Franck, Dorthe Rejkjær Holm, Kristian Schønning. (2019). Children Attending Day Care Centers are a Year-round Reservoir of Gastrointestinal Viruses. Sci Rep, vol. 9 (1). doi:10.1038/s41598-019-40077-9.

16. ^ Giovanna Russelli, Paola Pizzillo, Gioacchin Iannolo, Floriana Barbera, Fabio Tuzzolino, Rosa Liotta. (2017). HCV replication in gastrointestinal mucosa: Potential extra-hepatic viral reservoir and possible role in HCV infection recurrence after liver transplantation. PLOS ONE, vol. 12 (7), e0181683. doi:10.1371/journal.pone.0181683.

17. ^ Bin Zhang, Shuyi Liu, Yuhao Dong, Lu Zhang, Qingyang Zhong, Yujian Zou. (2020). Positive rectal swabs in young patients recovered from coronavirus disease 2019 (COVID-19). Journal of Infection. doi:10.1016/j.jinf.2020.04.023.

18. ^ Linlin Bao, Wei Deng, Hong Gao, Chong Xiao, Jiayi Liu, Jing Xue. (2020). Reinfection could not occur in SARS-CoV-2 infected rhesus macaques. doi:10.1101/2020.03.13.990226. 